(c) 2010 IEEE. Personal use of this material is permitted. Permission from IEEE must be obtained for all other uses, in any current or future media, including reprinting/republishing this material for advertising or promotional purposes, creating new collective works, for resale or redistribution to servers or lists, or reuse of any copyrighted component of this work in other works. 


\title{
Non-Regenerative Multi-Hop MIMO Relays Using MMSE-DFE Technique
}

\author{
Yue Rong \\ Department of Electrical and Computer Engineering, Curtin University of Technology, Bentley, WA 6102, Australia
}

\begin{abstract}
In this paper, we study multi-hop non-regenerative multiple-input multiple-output (MIMO) relay communications with any number of hops. We design the optimal source precoding matrix and the optimal relay amplifying matrices for such relay network where a nonlinear decision feedback equalizer (DFE) based on the minimal mean-squared error (MMSE) criterion is used at the destination node. We show that when the composite objective function is Schur-convex, the MMSE-DFE receiver together with the optimal source and relay matrices enable an arbitrary number of source symbols to be transmitted at one time, and yield a significantly improved performance compared with non-regenerative MIMO relay systems using linear receivers at the destination.
\end{abstract}

\section{INTRODUCTION}

Multi-hop relay communication is well known for being a cost-effective approach in improving the energy-efficiency of communication system in the case of long source-destination distance [1]. When nodes in the relay network are equipped with multiple antennas, we call such system a multiple-input multiple-output (MIMO) relay system. For a basic threenode two-hop MIMO relay system, optimal algorithms are developed in [2]-[5].

Recently, serially configured multi-hop MIMO relay communications with any number of hops attracted much research interest [6]-[10]. The asymptotic capacity of such system was studied in [6]-[8]. In [9], the authors investigated the diversity gain of multi-hop MIMO relay channel when the relays use diagonal amplifying matrices. Assuming that a linear minimal mean-squared error (MMSE) receiver is used at the destination, the MIMO relay design issue for most commonly used objective functions is investigated in [10].

It is well-known that a nonlinear decision feedback equalizer (DFE) has a much better bit-error-rate (BER) and mutual information (MI) performance than linear receivers [11]. In this paper, we apply the DFE receiver at the destination of a non-regenerative multi-hop MIMO relay system. In particular, the MMSE technique is applied to recover the source signal at each layer. We refer to such receiver as the MMSE-DFE receiver. We assume that the multi-hop MIMO relay link being considered has already been established by protocols at higher layers (link and/or network layers) [12]. We also assume that when a link failure is detected, the failed node can be bypassed by increasing the transmission power of the previous relay node, see [13] and the references therein. By applying the majorization theory [14] and the recently developed matrix generalized triangular decomposition (GTD) tool [15], we establish the structure of the optimal source precoding matrix and the optimal relay amplifying matrices for most commonly used objective functions.

Interestingly, we show that when the composite objective function is Schur-convex [14], the MMSE-DFE receiver together with the optimal source and relay matrices enable an arbitrary number of source symbols to be transmitted at one time, and the multi-hop MIMO relay channel is uniformly decomposed into identical subchannels. Numerical examples demonstrate that in such case, the MIMO relay system with a nonlinear MMSE-DFE receiver yields a significantly lower BER compared with a MIMO relay system using a linear receiver at the destination. We also show that for Schurconcave [14] composite objective functions, the optimal source and relay matrices, and the optimal feed-forward matrix at the destination node jointly diagonalize the multi-hop MIMO relay channel, and thus in such case, the nonlinear MMSE-DFE receiver is essentially equivalent to a linear MMSE receiver. Therefore, [10] can be treated as a special case of this paper where the composite objective function is Schur-concave. The relay scheme using the optimal source, relay matrices and the nonlinear MMSE-DFE receiver developed in this paper is more general than the relay scheme in [10].

We would like to mention that our paper extends the results in [16] from a single-hop MIMO channel to multi-hop nonregenerative MIMO relay communication systems with any number of hops. Note that the proof of the theorems for multihop MIMO relay system is much more involved than that for the single-hop MIMO channel. The extension from single-hop system to multi-hop system is significant, as the results are important for multi-hop wireless backhaul networks.

\section{SySTEM MODEL}

We consider a wireless communication system with one source node, one destination node, and $L-1$ relay nodes ( $L \geq 2$ ). We assume that due to the propagation path-loss, the signal transmitted by the $i$ th node can only be received by its direct forward node, i.e., the $(i+1)$-th node. Thus, signals transmitted by the source node pass through $L$ hops until they reach the destination node. We also assume that the number of antennas at each node is $N_{i}, i=1, \cdots, L+1$, and the number of source symbols in each transmission is $N_{b}$. Like [3]-[10], a linear non-regenerative relay matrix is used at each relay. However, in contrast to [3]-[10], a nonlinear MMSEDFE receiver is deployed at the destination node.

The $N_{1} \times 1$ signal vector transmitted by the source node is

$$
\mathbf{x}_{1}=\mathbf{F}_{1} \mathbf{s}
$$


where $\mathbf{s}=\left[s_{1}, s_{2}, \cdots, s_{N_{b}}\right]^{T}$ is the $N_{b} \times 1$ source symbol vector, $(\cdot)^{T}$ is the matrix (vector) transpose, and $\mathbf{F}_{1}$ is the $N_{1} \times$ $N_{b}$ source precoding matrix. We assume that $\mathrm{E}\left[\mathbf{s s}^{H}\right]=\mathbf{I}_{N_{b}}$, where $\mathrm{E}[\cdot]$ stands for the statistical expectation, $(\cdot)^{H}$ denotes the Hermitian transpose, and $\mathbf{I}_{n}$ is an $n \times n$ identity matrix. The $N_{i} \times 1$ signal vector received at the $i$ th node is written as

$$
\mathbf{y}_{i}=\mathbf{H}_{i-1} \mathbf{x}_{i-1}+\mathbf{v}_{i}, \quad i=2, \cdots, L+1
$$

where $\mathbf{H}_{i-1}$ is the $N_{i} \times N_{i-1}$ MIMO channel matrix between the $i$ th and the $(i-1)$-th nodes (i.e., the $(i-1)$-th hop), $\mathbf{v}_{i}$ is the $N_{i} \times 1$ i.i.d. additive white Gaussian noise (AWGN) vector at the $i$ th node with zero mean and unit variance, and $\mathbf{x}_{i-1}$ is the $N_{i-1} \times 1$ signal vector transmitted by the $(i-1)$-th node.

Using the linear non-regenerative strategy, the input-output relationship at node $i$ is given by

$$
\mathbf{x}_{i}=\mathbf{F}_{i} \mathbf{y}_{i}, \quad i=2, \cdots, L
$$

where $\mathbf{F}_{i}$ is the $N_{i} \times N_{i}$ relay amplifying matrix at node $i$. Combining (1)-(3), the received signal vector at the destination node (the $(L+1)$-th node) can be written as

$$
\mathbf{y}_{L+1}=\overline{\mathbf{H}} \mathbf{s}+\overline{\mathbf{v}}
$$

where $\overline{\mathbf{H}}$ and $\overline{\mathbf{v}}$ are the equivalent MIMO channel matrix and the equivalent noise vector, and given respectively by

$$
\begin{aligned}
\overline{\mathbf{H}} & =\bigotimes_{i=L}^{1}\left(\mathbf{H}_{i} \mathbf{F}_{i}\right) \\
\overline{\mathbf{v}} & =\sum_{l=2}^{L}\left(\bigotimes_{i=L}^{l}\left(\mathbf{H}_{i} \mathbf{F}_{i}\right) \mathbf{v}_{l}\right)+\mathbf{v}_{L+1} .
\end{aligned}
$$

Here for matrices $\mathbf{A}_{i}, \bigotimes_{i=l}^{k}\left(\mathbf{A}_{i}\right) \triangleq \mathbf{A}_{l} \cdots \mathbf{A}_{k}$. From (5) we see that $\overline{\mathbf{v}}$ is nonwhite with the following covariance matrix

$$
\mathbf{C}_{\bar{v}}=\sum_{l=2}^{L}\left(\bigotimes_{i=L}^{l}\left(\mathbf{H}_{i} \mathbf{F}_{i}\right) \bigotimes_{i=l}^{L}\left(\mathbf{F}_{i}^{H} \mathbf{H}_{i}^{H}\right)\right)+\mathbf{I}_{N_{L+1}} .
$$

At the destination node, a nonlinear DFE receiver is used to detect the source symbols successively with the $N_{b}$ th symbol detected first and the first symbol detected last. Assuming that there is no error propagation in the DFE receiver, the $k$ th source symbol is estimated as [11, Ch.8]

$$
\hat{s}_{k}=\mathbf{w}_{k}^{H} \mathbf{y}_{L+1}-\sum_{l=k+1}^{N_{b}} b_{k, l} s_{l}, \quad k=1, \cdots, N_{b} .
$$

It will be seen in Section IV that the error propagation effect only slightly increases the system BER. By introducing $\mathbf{W}=$ $\left[\mathbf{w}_{1}, \mathbf{w}_{2}, \cdots, \mathbf{w}_{N_{b}}\right], \hat{\mathbf{s}}=\left[\hat{s}_{1}, \hat{s}_{2}, \cdots, \hat{s}_{N_{b}}\right]^{T}$, and an $N_{b} \times N_{b}$ strictly upper-triangle matrix $\mathbf{B}$ with nonzero elements $b_{k, l}$, we can represent (7) in matrix form as

$$
\hat{\mathbf{s}}=\mathbf{W}^{H} \mathbf{y}_{L+1}-\mathbf{B} \mathbf{s}=\left(\mathbf{W}^{H} \overline{\mathbf{H}}-\mathbf{B}\right) \mathbf{s}+\mathbf{W}^{H} \overline{\mathbf{v}} .
$$

Here $\mathbf{W}$ and $\mathbf{B}$ are the feed-forward and feedback matrix of the DFE receiver, respectively. From (8) we find that the optimal $\mathbf{B}$ is given by

$$
\mathbf{B}=\mathcal{U}\left[\mathbf{W}^{H} \overline{\mathbf{H}}\right]
$$

where $\mathcal{U}[\mathbf{A}]$ denotes the strictly upper-triangular part of $\mathbf{A}$. Substituting (9) back into (7), we obtain

$$
\hat{s}_{k}=\mathbf{w}_{k}^{H}\left([\overline{\mathbf{H}}]_{1: k}[\mathbf{s}]_{1: k}+\overline{\mathbf{v}}\right), \quad k=1, \cdots, N_{b}
$$

where $[\mathbf{a}]_{1: k}$ denotes a vector containing the first $k$ elements of vector $\mathbf{a},[\mathbf{A}]_{1: k}$ stands for a matrix containing the first $k$ columns of $\mathbf{A}$.

When the MMSE criterion is used to estimate each symbol, from (10) the feed-forward matrix $\mathbf{W}$ is given by [11, Ch.8]

$$
\mathbf{w}_{k}=\left([\overline{\mathbf{H}}]_{1: k}[\overline{\mathbf{H}}]_{1: k}^{H}+\mathbf{C}_{\bar{v}}\right)^{-1} \overline{\mathbf{h}}_{k}, \quad k=1, \cdots, N_{b}
$$

where $\overline{\mathbf{h}}_{k}$ is the $k$ th column vector of $\overline{\mathbf{H}}$ and $(\cdot)^{-1}$ denotes the matrix inversion. Let us introduce the following $\mathrm{QR}$ decomposition [17]

$$
\mathbf{G} \triangleq\left[\begin{array}{c}
\mathbf{C}_{\overline{\mathbf{I}}}^{-\frac{1}{2}} \overline{\mathbf{H}} \\
\mathbf{I}_{N_{b}}
\end{array}\right]=\mathbf{Q R}=\left[\begin{array}{c}
\overline{\mathbf{Q}} \\
\mathbf{Q}
\end{array}\right] \mathbf{R}
$$

where $\mathbf{R}$ is an $N_{b} \times N_{b}$ upper-triangular matrix, $\mathbf{Q}$ is an $\left(N_{b}+\right.$ $\left.N_{L+1}\right) \times N_{b}$ semi-unitary matrix with $\mathbf{Q}^{H} \mathbf{Q}=\mathbf{I}_{N_{b}}, \overline{\mathbf{Q}}$ is a matrix containing the first $N_{L+1}$ rows of $\mathbf{Q}$, and $\mathbf{Q}$ contains the last $N_{b}$ rows of $\mathbf{Q}$. Obviously, we have from (12) that

$$
\mathbf{C}_{\bar{v}}^{-\frac{1}{2}} \overline{\mathbf{H}}=\overline{\mathbf{Q}} \mathbf{R}, \quad \underline{\mathbf{Q}}=\mathbf{R}^{-1} .
$$

Using (12) and (13), we can express $\mathbf{W}, \mathbf{B}$, and the MSE matrix of the signal waveform estimation $\mathbf{E}=$ $\mathrm{E}\left[(\hat{\mathbf{s}}-\mathbf{s})(\hat{\mathbf{s}}-\mathbf{s})^{H}\right]$ as follows.

THEOREM 1: Using the QR decomposition (12), W, B, and E can be written as

$$
\mathbf{W}=\mathbf{C}_{\bar{v}}^{-\frac{1}{2}} \overline{\mathbf{Q}} \mathbf{D}_{R}^{-1}, \quad \mathbf{B}=\mathbf{D}_{R}^{-1} \mathbf{R}-\mathbf{I}_{N_{b}}, \quad \mathbf{E}=\mathbf{D}_{R}^{-2}
$$

where $\mathbf{D}_{R}$ is a matrix taking the diagonal elements of $\mathbf{R}$ as the main diagonal and zero elsewhere.

Theorem 1 can be proven similar to [16, Lemma 4.1]. It is worth noting that Theorem 1 extends [16, Lemma 4.1] from white $\overline{\mathbf{v}}$ to nonwhite $\overline{\mathbf{v}}$. Interestingly, from (14) we find that for non-regenerative MIMO relay systems using the nonlinear MMSE-DFE receiver at the destination, $\mathbf{E}$ is diagonal for any channel realization.

\section{Optimal Source And Relay Matrices}

It has been shown in [10], [16] that most commonly used objective functions for MIMO systems can be represented as functions of the main diagonal elements of $\mathbf{E}$. It can be seen from (4), (6), and (12) that $\mathbf{D}_{R}$ depends on $\mathbf{F}_{i}, i=1, \cdots, L$. Thus, the source precoding matrix and all relay amplifying matrices need to be appropriately designed in order to optimize $q\left(\mathbf{d}\left[\mathbf{D}_{R}^{-2}\right]\right)$. Note that for a multi-hop single-input singleoutput (SISO) relay network [18] and serially configured sensor network [19], the generalized SNR objective function depends only on the last hop relay function, when the estimateand-forward relay protocol is used. Therefore, the multi-hop non-regenerative MIMO relay optimization problem is much more challenging than the SISO relay design problem. Using 
Theorem 1, the non-regenerative MIMO relay design problem can be formulated as

$$
\begin{aligned}
& \min _{\mathbf{D}_{R},\left\{\mathbf{F}_{i}\right\}} q\left(\mathbf{d}\left[\mathbf{D}_{R}^{-2}\right]\right) \\
& \text { s.t. }\left[\begin{array}{c}
\mathbf{C}_{\bar{v}}^{-\frac{1}{2}} \overline{\mathbf{H}} \\
\mathbf{I}_{N_{b}}
\end{array}\right]=\mathbf{Q R} \\
& \operatorname{tr}\left(\mathbf{F}_{1} \mathbf{F}_{1}^{H}\right) \leq p_{1} \\
& \operatorname{tr}\left(\mathbf { F } _ { i } \left(\sum_{l=1}^{i-1}\left(\bigotimes_{k=i-1}^{l}\left(\mathbf{H}_{k} \mathbf{F}_{k}\right) \bigotimes_{k=l}^{i-1}\left(\mathbf{F}_{k}^{H} \mathbf{H}_{k}^{H}\right)\right)\right.\right. \\
& \left.\left.+\mathbf{I}_{N_{i}}\right) \mathbf{F}_{i}^{H}\right) \leq p_{i}, \quad i=2, \cdots, L
\end{aligned}
$$

where $\left\{\mathbf{F}_{i}\right\} \triangleq\left\{\mathbf{F}_{i}, i=1, \cdots, L\right\}, q(\mathbf{x})$ stands for a unified objective function which is increasing with respect to each element of $\mathbf{x}, p_{i}>0$ is the transmission power available at node $i, \operatorname{tr}(\cdot)$ denotes the trace of a matrix, and for a matrix $\mathbf{A}, \mathbf{d}[\mathbf{A}]$ is a column vector containing all main diagonal elements of A. Here (17) and (18) are the transmission power constraints at the source node and all relay nodes, respectively.

Let us write the singular value decomposition (SVD) of $\mathbf{H}_{i}$ as

$$
\mathbf{H}_{i}=\mathbf{U}_{i} \boldsymbol{\Sigma}_{i} \mathbf{V}_{i}^{H}, \quad i=1, \cdots, L
$$

where the dimensions of $\mathbf{U}_{i}, \boldsymbol{\Sigma}_{i}, \mathbf{V}_{i}$ are $N_{i+1} \times N_{i+1}$, $N_{i+1} \times N_{i}, N_{i} \times N_{i}$, respectively. We assume that the main diagonal elements of $\boldsymbol{\Sigma}_{i}, i=1, \cdots, L$, are arranged in the decreasing order. We also introduce $M=\min \left(R_{h}, N_{b}\right)$, where $R_{h} \triangleq \min \left(\operatorname{rank}\left(\mathbf{H}_{1}\right), \operatorname{rank}\left(\mathbf{H}_{2}\right), \cdots, \operatorname{rank}\left(\mathbf{H}_{L}\right)\right)$ and $\operatorname{rank}(\cdot)$ denotes the rank of a matrix. The following theorem establishes the structure of the optimal source and relay matrices.

THEOREM 2: For multi-hop non-regenerative MIMO relay systems using nonlinear MMSE-DFE receiver at the destination, assuming that $\operatorname{rank}\left(\mathbf{F}_{i}\right)=M, i=1, \cdots, L$, the optimal source precoding matrix and the relay amplifying matrices are given by

$$
\mathbf{F}_{1}=\mathbf{V}_{1,1} \boldsymbol{\Lambda}_{1} \mathbf{V}_{F_{1}}^{H}, \quad \mathbf{F}_{i}=\mathbf{V}_{i, 1} \boldsymbol{\Lambda}_{i} \mathbf{U}_{i-1,1}^{H}, i=2, \cdots, L
$$

where $\boldsymbol{\Lambda}_{i}=\operatorname{diag}\left(\lambda_{i, 1}, \lambda_{i, 2}, \cdots, \lambda_{i, M}\right)$ are $M \times M$ diagonal matrices, $\mathbf{U}_{i, 1}$ and $\mathbf{V}_{i, 1}$ contain the leftmost $M$ vectors of $\mathbf{U}_{i}$ and $\mathbf{V}_{i}$, respectively, and $\mathbf{V}_{F_{1}}$ is an $N_{b} \times M$ semi-unitary matrix $\left(\mathbf{V}_{F_{1}}^{H} \mathbf{V}_{F_{1}}=\mathbf{I}_{M}\right)$ such that the $\mathrm{QR}$ decomposition in (16) holds with $\mathbf{D}_{R}$ being the solution to the problem (21)-(24) given below. In particular, $\left\langle\lambda_{i, k}\right\rangle \triangleq\left\{\lambda_{i, k}, 1 \leq i \leq L, 1 \leq k \leq\right.$ $M\}$ are obtained from the following optimization problem

$$
\begin{aligned}
& \min _{\mathbf{d}\left[\mathbf{D}_{R}\right],\left\langle\lambda_{i, k}\right\rangle} q\left(\mathbf{d}\left[\mathbf{D}_{R}^{-2}\right]\right) \\
& \text { s.t. } \mathbf{d}\left[\mathbf{D}_{R}^{2}\right] \prec \times(\mathrm{w}) \mathbf{g} \\
& \sum_{k=1}^{M} \lambda_{1, k}^{2} \leq p_{1} \\
& \sum_{k=1}^{M} \lambda_{i, k}^{2}\left(\sum_{j=1}^{i-1} \prod_{l=j}^{i-1} \lambda_{l, k}^{2} \sigma_{l, k}^{2}+1\right) \leq p_{i}, i=2, \cdots, L
\end{aligned}
$$

where $\sigma_{i, k}$ is the $k$ th main diagonal element of $\boldsymbol{\Sigma}_{i}, \prec_{x}$ (w) stands for weakly multiplicative submajorization [14], and $\mathbf{g} \triangleq$ $\left[\left\{1+\frac{\prod_{i=1}^{L} \sigma_{i, k}^{2} \lambda_{i, k}^{2}}{1+\sum_{i=2}^{L} \prod_{l=i}^{L} \sigma_{l, k}^{2} \lambda_{l, k}^{2}}\right\}_{M}^{T}, \mathbf{1}_{N_{b}-M}\right]^{T}$. Here for a scalar $x,\{x\}_{n} \triangleq\left[x_{1}, x_{2}, \cdots, x_{n}\right]^{T}$, and $\mathbf{1}_{n}$ denotes a $1 \times n$ vector with all 1 elements.

PROOF: See the journal version of this paper [20].

The motivation of the assumption $\operatorname{rank}\left(\mathbf{F}_{i}\right)=M$ is to avoid any transmission power loss at each node. It is worth noting that Theorem 2 simplifies the matrix-variable optimization problem (15)-(18) to the problem (21)-(24) with scalar variables. Moreover, (20) presents the structure of the optimal $\mathbf{F}_{i}$ in the form of its singular value decomposition. For $i=2, \cdots, L$, the left and right singular vector matrices of $\mathbf{F}_{i}$, (i.e., $\mathbf{V}_{i, 1}$ and $\mathbf{U}_{i-1,1}$ ) are known, while the singular value matrix $\boldsymbol{\Lambda}_{i}$ is obtained by solving the problem (21)-(24). For the optimal $\mathbf{F}_{1}, \mathbf{V}_{1,1}$ is known, $\boldsymbol{\Lambda}_{1}$ is obtained from (21)-(24), while $\mathbf{V}_{F, 1}$ is chosen to guarantee that the $\mathrm{QR}$ decomposition (12) yields the $\mathbf{D}_{R}$ in (21). In particular, we carry out the GTD [15] of the following matrix

$$
\begin{aligned}
\boldsymbol{\Psi} & \triangleq\left[\begin{array}{c}
\left.\mathbf{C}_{\bar{v}}^{-\frac{1}{2}} \bigotimes_{i=L}^{2}\left(\mathbf{H}_{i} \mathbf{F}_{i}\right) \mathbf{H}_{1} \mathbf{V}_{1,1}\left[\boldsymbol{\Lambda}_{1}, \mathbf{0}_{M \times\left(N_{b}-M\right)}\right]\right] \\
\mathbf{I}_{N_{b}}
\end{array}\right. \\
& =\mathbf{Q}_{\Psi} \mathbf{R P}_{\Psi}^{H}
\end{aligned}
$$

where $\mathbf{R}$ has the diagonal elements $\mathbf{D}_{R}$ obtained from the first step, and $\mathbf{0}_{p \times m}$ denotes a $p \times m$ matrix with all zero entries. Then we obtain $\mathbf{V}_{F_{1}}=\left[\mathbf{P}_{\Psi}^{H}\right]_{1: M}$ and consequently $\mathbf{F}_{1}$ in (20). The justification of the second step can be found in [20].

For general objective functions, the problem (21)-(24) is still complicated, especially the constraint (22). Let us define the composite objective function of $q \diamond \exp (\mathbf{x}) \triangleq$ $q\left(e^{x_{1}}, e^{x_{2}}, \cdots, e^{x_{N_{b}}}\right)$. In the following, we show that for Schur-concave $q \diamond \exp$ and Schur-convex $q \diamond \exp$, the problem (21)-(24) can be further simplified. Examples of Schur-convex $q \diamond$ exp can be found in [16] such as the sum of MSEs of all data streams, which can be expressed as $\sum_{k=1}^{N_{b}}\left[\mathbf{D}_{R}\right]_{k, k}^{-2}$ using (14). Some Schur-concave $q \diamond \exp$ are also listed in [16] such as the exponentially weighted product of MSEs which is written as $\prod_{k=1}^{N_{b}}\left[\mathbf{D}_{R}\right]_{k, k}^{-2 \alpha_{k}}$, where $0<\alpha_{1} \leq \cdots \leq \alpha_{N_{b}}$ are the weighting coefficients.

THEOREM 3: For the relay design problem (21)-(24), if $q \diamond$ exp is increasing and Schur-concave, then the optimal $\mathbf{V}_{F_{1}}$ is equal to $\left[\mathbf{I}_{M}, \mathbf{0}_{M \times\left(N_{b}-M\right)}\right]^{T}$, and $\left\langle\lambda_{i, k}\right\rangle$ is the solution to the following problem

$$
\begin{aligned}
\min _{\left\langle\lambda_{i, k}\right\rangle} & q(\mathbf{g}) \\
\text { s.t. } & \sum_{k=1}^{M} \lambda_{1, k}^{2} \leq p_{1} \\
& \sum_{k=1}^{M} \lambda_{i, k}^{2}\left(\sum_{j=1}^{i-1} \prod_{l=j}^{i-1} \lambda_{l, k}^{2} \sigma_{l, k}^{2}+1\right) \leq p_{i}, \quad i=2, \cdots, L .
\end{aligned}
$$

PROOF: See the journal version of this paper [20]. 
Interestingly, we find that for Schur-concave $q \diamond \exp$, without wasting the transmission power at the source node, the number of symbols $N_{b}$ should be no greater than $R_{h}$.

Assuming that $N_{b}=R_{h}=M$ and substituting (20) along with $\mathbf{V}_{F_{1}}=\mathbf{I}_{N_{b}}$ back into (4) and (6), we obtain

$$
\begin{aligned}
\overline{\mathbf{H}} & =\mathbf{U}_{L, 1} \mathbf{D}_{h} \\
\mathbf{C}_{\bar{v}} & =\mathbf{U}_{L, 1} \mathbf{D}_{c} \mathbf{U}_{L, 1}^{H}+\mathbf{I}_{N_{L+1}}
\end{aligned}
$$

where $\mathbf{D}_{h}$ and $\mathbf{D}_{c}$ are $N_{b} \times N_{b}$ diagonal matrices with the $k$ th diagonal elements $k=1, \cdots, N_{b}$, given by $d_{h, k}=$ $\prod_{l=1}^{L} \lambda_{l, k} \sigma_{l, k}$ and $d_{c, k}=\sum_{l=2}^{L} \prod_{i=l}^{L} \lambda_{i, k}^{2} \sigma_{i, k}^{2}$, respectively. From (28) we have for $k=1, \cdots, N_{b}$

$$
\overline{\mathbf{h}}_{k}=d_{h, k} \mathbf{u}_{k}, \quad[\overline{\mathbf{H}}]_{1: k}=\left[\mathbf{U}_{L, 1}\right]_{1: k}\left[\mathbf{D}_{h}\right]_{1: k, 1: k}
$$

where $\mathbf{u}_{k}$ is the $k$ th column vector of $\mathbf{U}_{L, 1}$, and for any matrix $\mathbf{A},[\mathbf{A}]_{1: k, 1: k}$ is a principal sub-matrix of $\mathbf{A}$ lying in the first $k$ rows and the first $k$ columns of A. Substituting (29) and (30) back into (11), we have

$$
\mathbf{w}_{k}=\frac{d_{h, k}}{d_{h, k}^{2}+d_{c, k}+1} \mathbf{u}_{k}
$$

Thus, the feed-forward matrix can be represented as $\mathbf{W}=$ $\mathbf{U}_{L, 1} \mathbf{D}_{w}$, where $\mathbf{D}_{w}$ is an $N_{b} \times N_{b}$ diagonal matrix with the $k$ th diagonal element given by $d_{w, k}=d_{h, k} /\left(d_{h, k}^{2}+d_{c, k}+1\right)$. Now we have $\mathbf{W}^{H} \overline{\mathbf{H}}=\mathbf{D}_{w} \mathbf{U}_{L, 1}^{H} \mathbf{U}_{L, 1} \mathbf{D}_{h}=\mathbf{D}_{w} \mathbf{D}_{h}$ which is an $N_{b} \times N_{b}$ diagonal matrix with the $k$ th diagonal element as $d_{h, k}^{2} /\left(d_{h, k}^{2}+d_{c, k}+1\right)$. This indicates that when $q \diamond \exp$ is Schur-concave, the optimal source precoding matrix (20) along with $\mathbf{V}_{F_{1}}=\mathbf{I}_{N_{b}}$, the optimal relay amplifying matrices (20), and the optimal feed-forward matrix at the destination (31) jointly diagonalize the multi-hop MIMO relay channel. From (9), we find that the feedback matrix $\mathbf{B}=\mathbf{0}_{N_{b} \times N_{b}}$. Thus, the nonlinear MMSE-DFE receiver is identical to a linear MMSE receiver.

THEOREM 4: For the relay design problem (21)-(24), if $q \diamond$ exp is increasing and Schur-convex, then the optimal $\mathbf{V}_{F_{1}}$ is chosen such that $\mathbf{d}\left[\mathbf{D}_{R}\right]$ has equal entries, and $\left\langle\lambda_{i, k}\right\rangle$ can be obtained from

$$
\begin{aligned}
\min _{\left\langle\lambda_{i, k}\right\rangle} & -\sum_{k=1}^{M} \log \left(1+\frac{\prod_{i=1}^{L} \sigma_{i, k}^{2} \lambda_{i, k}^{2}}{1+\sum_{i=2}^{L} \prod_{l=i}^{L} \sigma_{l, k}^{2} \lambda_{l, k}^{2}}\right) \\
\text { s.t. } & \sum_{k=1}^{M} \lambda_{1, k}^{2} \leq p_{1} \\
& \sum_{k=1}^{M} \lambda_{i, k}^{2}\left(\sum_{j=1}^{i-1} \prod_{l=j}^{i-1} \lambda_{l, k}^{2} \sigma_{l, k}^{2}+1\right) \leq p_{i}, \quad i=2, \cdots, L .
\end{aligned}
$$

PROOF: See the journal version of this paper [20].

It can be seen from [20] that for Schur-convex $q \diamond \exp$, $\mathbf{d}\left[\mathbf{D}_{R}\right]$ has identical elements. Thus, the multi-hop MIMO relay channel is uniformly decomposed into $N_{b}$ identical subchannels. In contrast to the case of Schur-concave $q \diamond \exp$, there is no constraint on $N_{b}$. In fact, $N_{b}$ can be greater than
$R_{h}$ for Schur-convex $q \diamond$ exp. Substituting (20) back into (4) and (6), we obtain $\mathbf{C}_{\bar{v}}$ in (29) and

$$
\overline{\mathbf{H}}=\mathbf{U}_{L, 1} \mathbf{D}_{h} \mathbf{V}_{F_{1}}^{H} .
$$

Substituting (29) and (35) back into (11) we have

$$
\begin{aligned}
\mathbf{w}_{k}^{H} \overline{\mathbf{h}}_{l}= & {\left[\mathbf{V}_{F_{1}}^{H}\right]_{k}^{H} \mathbf{D}_{h}\left(\mathbf{D}_{h}\left[\mathbf{V}_{F_{1}}^{H}\right]_{1: k}\left[\mathbf{V}_{F_{1}}^{H}\right]_{1: k}^{H} \mathbf{D}_{h}+\mathbf{D}_{c}+\mathbf{I}_{M}\right)^{-1} } \\
& \times \mathbf{D}_{h}\left[\mathbf{V}_{F_{1}}^{H}\right]_{l} .
\end{aligned}
$$

We find from (36) that due to $\mathbf{V}_{F_{1}} \neq\left[\mathbf{I}_{M}, \mathbf{0}_{M \times\left(N_{b}-M\right)}\right]^{T}$, $\mathbf{w}_{k}^{H} \overline{\mathbf{h}}_{l} \neq 0$. Consequently, $\mathbf{W}^{H} \overline{\mathbf{H}}$ is not diagonal and from (9) $\mathbf{B} \neq \mathbf{0}_{N_{b} \times N_{b}}$. This is the reason that more data streams $N_{b}$ can be supported than the number of subchannels $R_{h}$. Thus, in this case, the MIMO relay system with nonlinear MMSEDFE receiver at the destination has a different performance compared with the MIMO relay system using the linear MMSE receiver at the destination. We will show through numerical simulations that the former system has a much better performance than the latter one.

After the structure of the optimal source and relay matrices is determined, the remaining problem is to optimize the power loading vector $\left\langle\lambda_{i, k}\right\rangle$ by solving the problem (25)-(27) and the problem (32)-(34) for Schur-concave and Schur-convex $q \diamond \exp$, respectively. Unfortunately, for $L \geq 2$, both problems are nonconvex. Hence, a globally optimal solution is difficult to obtain with affordable computational complexity, especially when $L$ is large. However, a locally optimal solution can be obtained by iteratively updating the power allocation vector at one node by fixing the power allocation vectors at all other nodes [10]. This iterative algorithm provides an excellent performance-complexity tradeoff. Finally, for the MIMO relay design problem with Schur-convex $q \diamond \exp$, matrix $\mathbf{V}_{F_{1}}$ can be computed using the numerical method developed in [15].

\section{NUMERICAL EXAMPLES}

In the simulations, all channel matrices have complex Gaussian entries with zero-mean and variances $\sigma_{i}^{2} / N_{i}$ for $\mathbf{H}_{i}$, $i=1, \cdots, L$. Consequently, we define $\mathrm{SNR}_{i} \triangleq \sigma_{i}^{2} p_{i} N_{i+1} / N_{i}$ as the signal-to-noise ratio (SNR) of the $i$ th hop. All results are averaged over 1000 independent channel realizations.

We compare the relay algorithm for Schur-convex $q \diamond \exp$ (denoted as the NL (nonlinear) algorithm) with the maximal MI (MMI) algorithm and the MMSE algorithm developed in [10]. The MMI algorithm refers to a multi-hop MIMO relay system with a linear MMSE receiver at the destination node, and the source and relay matrices designed to maximize the source-destination MI. Thus, both the MMI and the MMSE algorithms apply the linear MMSE receiver at the destination. The comparison is fair since the MMI and MMSE relay algorithms in [10] and the proposed NL algorithm require the same amount of channel information at each node. Moreover, all algorithms use the iterative approach to obtain the optimal power allocation vectors, and thus, have the same computational complexity order. As a benchmark, we also show the performance of the genie-aided NL algorithm, where at each layer the error propagation is eliminated by a genie. 


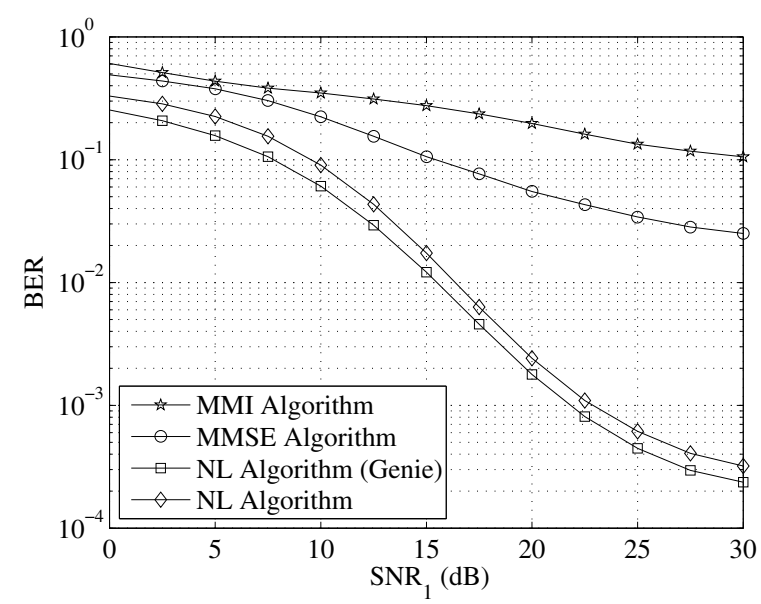

Fig. 1. Two hops. $N_{1}=N_{2}=N_{3}=3, N_{b}=3, \mathrm{SNR}_{2}=20 \mathrm{~dB}$.

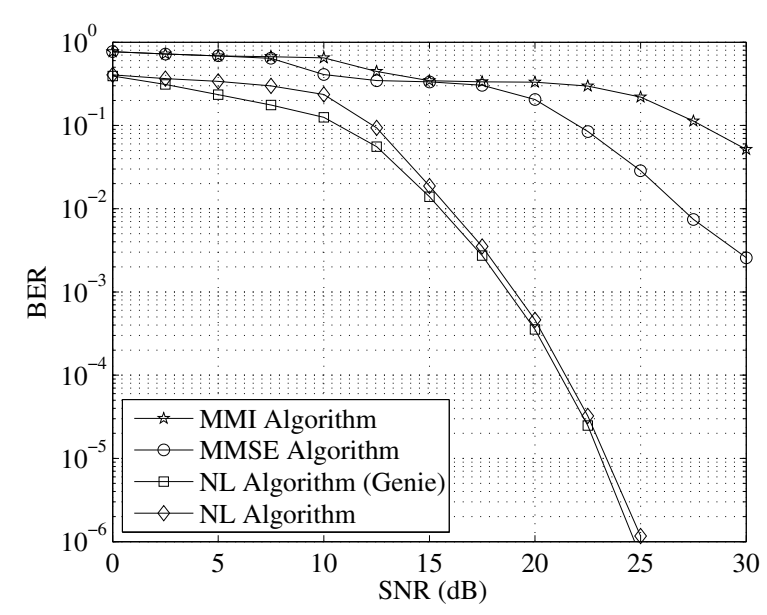

Fig. 2. Five hops. $N_{i}=3, i=1, \cdots, 6, \mathrm{SNR}_{i}=\mathrm{SNR}, i=1, \cdots, 5$.

In the first example, we simulate a fully loaded two-hop system with $N_{1}=N_{2}=N_{3}=3, N_{b}=3$, and $\mathrm{SNR}_{2}=20 \mathrm{~dB}$. The performance of all algorithms is shown in Fig. 1. It can be seen that the proposed NL algorithm performs consistently better than the competing algorithms over the whole $\mathrm{SNR}_{1}$ range. In fact, the nonlinear MMSE-DFE receiver achieves a much higher diversity order than the linear receiver.

In the second example, a multi-hop MIMO relay system with $L=5$ and $N_{i}=3, i=1, \cdots, 6$ is simulated. Each hop has the same SNR, i.e, $\mathrm{SNR}_{i}=\mathrm{SNR}, i=1, \cdots, 5$. Fig. 2 displays the BER performance of all three algorithms versus SNR. Obviously, for multi-hop systems, the NL algorithm still has the best performance. Therefore, for multihop MIMO relay communication, the nonlinear MMSE-DFE receiver together with the optimal source and relay matrices developed in this paper should be used in order to achieve a lower system BER. Comparing the practical NL algorithm with the genie-aided NL algorithm, we find from Figs. 1 and 2 that the propagation of the detection error at each layer only slightly increases the system BER.

\section{CONCLUSIONS}

We have developed the optimal source and relay matrices for non-regenerative multi-hop MIMO relay systems when the nonlinear MMSE-DFE receiver is used at the destination. For Schur-convex composite objective functions, the optimal scheme uniformly decomposes the MIMO relay channel into identical subchannels without any constraints on the number of subchannels. We have shown that the BER performance can be significantly improved by using the MMSE-DFE receiver.

\section{REFERENCES}

[1] M. Cao, X. Wang, S.-J. Kim, and M. Madihian, "Multi-hop wireless backhaul networks: A cross-layer design paradigm” IEEE J. Sel. Areas Commun., vol. 25, pp. 738-748, May 2007.

[2] B. Wang, J. Zhang, and A. Høst-Madsen, "On the capacity of MIMO relay channels," IEEE Trans. Inform. Theory, vol. 51, pp. 29-43, Jan. 2005.

[3] X. Tang and Y. Hua, "Optimal design of non-regenerative MIMO wireless relays," IEEE Trans. Wireless Commun., vol. 6, pp. 1398-1407, Apr. 2007.

[4] I. Hammerström and A. Wittneben, "Power allocation schemes for amplify-and-forward MIMO-OFDM relay links," IEEE Trans. Wireless Commun., vol. 6, pp. 2798-2802, Aug. 2007.

[5] A. S. Behbahani, R. Merched, and A. M. Eltawil, "Optimizations of a MIMO relay network," IEEE Trans. Signal Processing, vol. 56, pp. 5062-5073, Oct. 2008.

[6] S. Yeh and O. Lévêque, "Asymptotic capacity of multi-level amplifyand-forward relay networks," in Proc. IEEE ISIT, Nice, France, Jun. 2007, pp. 1436-1440.

[7] N. Fawaz, K. Zarifi, M. Debbah, and D. Gesbert, "Asymptotic capacity and optimal precoding strategy of multi-level precode \& forward in correlated channels," in Proc. IEEE ITW, Porto, Portugal, May 2008, pp. 209-213.

[8] J. Wagner and A. Wittneben, "On capacity scaling of (long) MIMO amplify-and-forward multi-hop networks," in Proc. IEEE Asilomar Conference on Signals, Systems and Computers, Pacific Grove, CA, USA, Oct. 2008, pp. 346-350.

[9] S. Yang and J.-C. Belfiore, "Diversity of MIMO multihop relay channels," submitted to IEEE Trans. Inform. Theory, [Online]. Avaliable: http://arxiv.org/abs/0708.0386v1.

[10] Y. Rong and Y. Hua, "Optimality of diagonalization of multi-hop MIMO relays," IEEE Trans. Wireless Commun., vol. 8, pp. 6068-6077, Dec. 2009.

[11] D. Tse and P. Viswanath, Fundamentals of Wireless Communication. Cambridge University Press, 2005.

[12] H. Alwan and A. Agarwal, "A survey on fault tolerant routing techniques in wireless sensor networks," in Proc. 3rd Int. Conf. Sensor Tech. Applications, Athens, Greece, Jun. 18-23, 2009, pp. 366-371.

[13] N. A. Pantazis and D. D. Vergados, "A survey on power control issues in wireless sensor networks," IEEE Commun. Surveys \& Tutorials, vol. 9, pp. 86-107, 4th Quarter, 2007.

[14] A. W. Marshall and I. Olkin, Inequalities: Theory of Majorization and Its Applications. Academic Press, 1979.

[15] Y. Jiang, W. Hager, and J. Li, "The generalized triangular decomposition," Mathematics of Computation, vol. 77, pp. 1037-1056, Apr. 2008.

[16] D. P. Palomar and Y. Jiang, MIMO Transceiver Design via Majorization Theory. now Publishers, 2007.

[17] R. A. Horn and C. R. Johnson, Matrix Analysis. Cambridge University Press, 1985.

[18] K. S. Gomadam and S. A. Jafar, "Optimal relay functionality for SNR maximization in memoryless relay networks," IEEE J. Select. Areas Commun., vol. 25, pp. 390-401, Feb. 2007.

[19] R. Viswanathan and P. K. Varshney, "Distributed detection with multiple sensors: Part I - Fundamentals," Proceeding of the IEEE, vol. 85, pp. 5463, Jan. 1997.

[20] Y. Rong, "Optimal linear non-regenerative multi-hop MIMO relays with MMSE-DFE Receiver at the destination," IEEE Trans. Wireless Commun., vol. 9, pp. 2268-2279, Jul. 2010. 